\title{
JUPITER RADIO FINE STRUCTURES OBSERVED IN DECAMETRIC FREQUENCY RANGE BY URAN-2 RADIO TELESCOPE
}

\author{
J. Schiemel*, M. Panchenko ${ }^{\dagger}$ H. O. Rucker*, A. I. Brazhenko \\ and A. A. Konovalenko ${ }^{\S}$
}

\begin{abstract}
We report the observations of fine radio structures in Jovian decametric radio emission. In our study we have used the data obtained by the large ground-based radio telescope URAN-2 during four observation campaigns from September 2012 to March 2016. The campaigns included long-term continuous observation of Jovian radio emission (during periods of Jupiter's visibility) in the frequency range 8$32 \mathrm{MHz}$, with time-frequency resolution $0.1 \mathrm{~s}$ and $4 \mathrm{kHz}$. A unique observational material has been obtained including many events of Io controlled DAM, non-Io DAM as well as fine spectral radio structures such as trains of S-bursts, quasicontinuous narrowband emissions, narrow-band splitting events and zebra stripelike patterns. We performed a preliminary statistical analysis of observations of narrow band events. Such parameters as occurrence in Central Meridian Longitude of Jupiter, intensity, duration of the events, frequency range and frequency drift have been defined. The first results are presented.
\end{abstract}

\footnotetext{
* Commission for Astronomy, Austrian Academy of Sciences, Graz, Austria

$\dagger$ Space Research Institute, Austrian Academy of Sciences, Graz, Austria

$\ddagger$ Institute of Geophysics, Gravimetric Observatory, Poltava, Ukraine

$\S$ Institute of Radio Astronomy, National Academy of Sciences of the Ukraine, Kharkov, Ukraine
} 
